\title{
Amossy, Ruth. 2014. Apologie de la polémique (Paris : PUF, coll. «L'interrogation philosophique »)
}

Loïc Nicolas

\section{OpenEdition}

1 Journals

Electronic version

URL: http://journals.openedition.org/aad/2057

DOI: $10.4000 /$ aad. 2057

ISSN: 1565-8961

Publisher

Université de Tel-Aviv

\section{Electronic reference}

Loïc Nicolas, «Amossy, Ruth. 2014. Apologie de la polémique (Paris : PUF, coll. «L'interrogation philosophique ») », Argumentation et Analyse du Discours [Online], 15 | 2015, Online since 15 October 2015, connection on 10 December 2020. URL : http://journals.openedition.org/aad/2057 ; DOI : https://doi.org/10.4000/aad.2057

This text was automatically generated on 10 December 2020.

\section{(c) (i) $\ominus$}

Argumentation \& analyse du discours est mis à disposition selon les termes de la licence Creative Commons Attribution - Pas d'Utilisation Commerciale - Pas de Modification 4.0 International. 


\title{
Amossy, Ruth. 2014. Apologie de la polémique (Paris : PUF, coll. "L'interrogation philosophique »)
}

\author{
Loïc Nicolas
}

\section{REFERENCES}

Amossy, Ruth. 2014. Apologie de la polémique (Paris : PUF, coll. « L'interrogation philosophique »), ISBN : 978-2-13-062440-0, 239 pages

1 Cette Apologie de la polémique, récemment parue dans la collection dirigée par Michel Meyer aux Presses Universitaires de France, constitue, sans nul doute, l'un des ouvrages les plus pénétrants de Ruth Amossy. De la polémique, l'auteur s'est d'ailleurs fait, depuis 2002, une spécialité (aux côtés de recherches sur l'ethos et le stéréotype), lui consacrant de nombreux travaux dont la liste est reprise en fin de volume (238-239). Évitant bien des écueils, tant méthodologiques qu'épistémologiques, l'enquête conduite par Amossy mêle, avec cohérence, synthèse et analyse ; entreprise théorique et étude de cas concrets. Elle s'emploie à rendre au dissensus (sa monstration, son exploration, sa conservation) ses lettres de noblesse. Elle en revisite le potentiel créatif, et signale dans quelle mesure l'expérience du désaccord contribue, de façon quelque peu paradoxale, à fonder la concorde sociale. La polémique, en tant que « phénomène global » (9), et tout spécialement la polémique dans sa dimension publique (relayée, alimentée par les médias), se voit présentée de façon réaliste comme « une modalité argumentative parmi d'autres ", et non pas comme «un genre de discours » délimité (70). Ses fonctions sociales et politiques, ses usages et modalités d'exercice, mais aussi ses ambiguïtés, frontières et paradoxes, font l'objet d'un traitement rigoureux qui donne de l'épaisseur à cet événement discursif qui fascine (en secret, en privé) autant qu'il rebute (en public). À vrai dire, la place spectaculaire - au plein sens du terme - occupée par la polémique dans l'espace médiatique (presse écrite, télévision, internet) contraste avec le mépris, revendiqué à hauts cris, dans lequel on la tient - et ceci, à l'intérieur comme 
à l'extérieur du champ scientifique. Vaine, puérile, ridicule, irrationnelle, infamante, odieuse, sans fondements, etc., les mots pour la disqualifier ne manquent pas. Personne (ou presque) ne semble disposé à lui trouver le moindre crédit; à lui reconnaître une valeur pratique.

2 Prenant acte de cette " contradiction flagrante » (8), l'auteur cherche à en détailler les raisons, et restaure, par touches successives, le caractère éminemment complexe, et presque déroutant, du fait polémique. Une complexité qu'ignorent allégrement les censeurs de tous bords; les philosophes rationalistes et idéalistes à la poursuite d'un logos entièrement purifié (Angenot 2008 : 56-57) ; les adeptes d'une approche normative de l'argumentation. Lesquels, sans nuance et de concert, se plaisent à blâmer ce type de discours au motif qu'il serait " partial et entaché de passion » autant que de violence (7) ; au motif qu'il contreviendrait aux règles (saines et froides) de la raison bien menée. Ainsi trahissent-ils, en premier lieu, leur peur du pluralisme, de la critique et du désaccord, en somme, leur peur du désordre des mots comme celui des hommes. Or, ce "désordre ", qu'on le veuille ou non; qu'on s'en offusque ; qu'on le refuse avec force, demeure, écrit Chaïm Perelman, la « rançon inévitable de la liberté » (2009 [1945] : 56). Dès lors, au fil des pages, Amossy soutient (contre la doxa impraticable des chantres de la bien-pensance et du logos désincarné) que « la polémique remplit des fonctions sociales importantes précisément en raison de ce qui lui est généralement reproché: une gestion verbale $d u$ conflit effectuée sur le mode $d u$ dissensus " (12, souligné par l'auteur). Cette thèse très forte, défendue avec beaucoup de conviction, est l'occasion d'aborder des questions cruciales relatives à la pratique de la délibération et au bon fonctionnement de la démocratie.

3 En trois parties et six chapitres équilibrés, Amossy se livre, dans un geste assumé, à une véritable apologie de la polémique, et s'efforce de réconcilier celle-ci avec la rhétorique; de "faire juste place à une rhétorique du dissensus» (42). Elle dénonce, pour ce faire, une conception étriquée de l'antique technè, définie, trop souvent, comme " recherche du consensus » à tout prix (12); comme " art de négocier les différences pour arriver à un accord» (19); comme manière «de diriger la cité grâce au partage d'une parole maîtrisée » (21). Ceci dit, cette représentation courante de la rhétorique est peut-être (sans doute, même) moins liée à ce qu'elle est vraiment qu'à une certaine écriture politique de son histoire. Une écriture orientée, qui n'a fait que l'éloigner de son «inscription corporelle et de sa dynamique actionnelle», la constituant en une entité « abstraite et figée, comme pour protéger le citoyen de la complexité du monde » (Danblon $2013: 26$ ).

4 Au reste, pour apologétique qu'il soit, le geste d'Amossy n'en est pas cynique pour autant. Il est humaniste et invite à une conversion positive du regard; à un changement de point de vue. Il prend acte de «la nature conflictuelle des démocraties contemporaines » (12), et reconnaît qu'elles sont marquées par l'extrême pluralité des conceptions du monde et de la vie bonne (eu zên); par la liberté d'expression; par l'affrontement des mots et des opinions. De fait, la polémique, cette façon d'être (donc de vivre) ensemble dans la confrontation verbale, témoigne de ce qu'il y a au plus profond de l'expérience démocratique - là où les citoyens pratiquent et exercent leur liberté. Elle rend tangible l'existence de "problèmes non susceptibles d'une solution considérée comme une vérité objective » (Perelman 1948:13). Des problèmes que nul ne saurait trancher de façon définitive, ni pour tout un chacun. Des problèmes qui relèvent du jugement pratique, non de la connaissance des choses. 
5 Dès l'introduction de l'ouvrage (7-13), le décor est planté ; l'idée phare est livrée sans détour, à savoir que la polémique, bien qu'elle ne permette pas «d'aboutir à un accord " (car là n'est pas sa fonction), constitue le moyen privilégié «d'assurer un mode de coexistence dans une communauté déchirée entre des positions et des intérêts divergents » (13). La "virulence » et les « excès » qui la caractérisent ne sont pas, comme on le croit d'ordinaire, un moins, un drame, une maladie du discours, ou une preuve de son irrationalité patente. Au contraire, par ces excès mêmes, s'expriment la capacité, et plus, la volonté des « participants de partager le même espace sans recourir à la violence physique» (13). Partant, la polémique est non seulement utile, fonctionnelle, mais elle occupe un rôle considérable, dans la structuration et l'organisation de l'espace politique, en particulier dans les démocraties que nous connaissons. Tout au long de son enquête, Amossy s'emploie à informer cette proposition, qui, bien qu'elle se situe dans la continuité directe de ses productions antérieures $(2010,2011)$, demeure très largement inédite, notamment dans sa formulation.

6 Les deux chapitres de la première partie (17-70) sont les plus théoriques du volume. L'auteur y aborde, d'une part, différentes approches de l'accord ou, plus précisément, du désaccord en démocratie (les craintes qu'il ne cesse d'inspirer; sa condamnation permanente ; sa possible revalorisation), d'autre part, certains aspects définitoires de la polémique. Amossy relève d'emblée combien les démocraties libérales, à cet égard assez fermement platoniciennes, sont hantées par l'accord: sa recherche, sa mise à jour, et surtout son triomphe. Un accord qui représente, tout à la fois, l'« objectif majeur de toute délibération bien comprise » (20), et le garant ultime d'une société harmonieuse. En se mettant d'accord; en nettoyant leurs propos de toute trace d'éristique, de véhémence, de passion, d'engagement, les interlocuteurs sont, de la sorte, supposés " obéir aux impératifs de la raison» (19).

7 Comprenons bien, le désaccord, lieu de mille fantasmes, n'en finit pas d'être regardé comme «le signe d'un échec» (27), et mieux, comme un risque; un péril à venir. La grande peur est de voir, par sa faute, «sombrer la communauté dans la discorde, la division, voire la lutte armée»(22). Raison pour laquelle, il convient, par tous les moyens, de le dénoncer; de le traquer; d'en dépouiller l'espace politique. Ce à quoi s'attachent justement les pourfendeurs de la joute oratoire; les défenseurs du débat entre partenaires policés - débat considéré comme l'idéal « de la communication dans l'espace public » (29). Il en va ainsi, à des titres divers, de Jürgen Habermas, des pragma-dialecticiens de l'École d'Amsterdam, des tenants de la logique dite informelle. Je me montrerai, toutefois, plus réservé quant à l'inclusion de Chaïm Perelman au sein de cet ensemble-là. Quoique certaines formulations du penseur belge puissent prêter à confusion, il n'y a, chez lui, aucune idéalisation de l'accord en tant que fin en soi, en tant qu'horizon espéré de l'échange argumentatif. On trouve, au contraire, dans son œuvre, une reconnaissance bien réelle de la valeur du désaccord (Perelman 1990 [1966], 1989 [1968], 1977). Qu'on pense ici, par exemple, à l'article majeur qu'il consacre au "problème du bon choix », où il précise :

Les désaccords que l'exercice de [la] liberté a provoqués parmi les hommes ont donné du prix à l'établissement d'une méthode rationnelle qui permettrait de fournir les critères incontestables $d u$ bon choix. Mais les succès partiels obtenus dans le domaine technique ne devraient pas faire oublier la part d'illusion qu'il y a à vouloir obtenir, à l'aide de méthodes scientifiques, un accord sur tous les problèmes de conduite : on risquerait, au nom du mythe de la «raison ", de transformer des 
consciences humaines en automates ajustés aux besoins d'une idéologie (Perelman

1952 [1948] : 160).

On ne saurait, je crois, être plus clair.

Amossy revient ensuite sur les efforts de "revalorisation du dissensus » (34) en s'appuyant, notamment, sur les travaux de Chantal Mouffe. Laquelle refuse de voir dans le consensus "la clé de la démocratie ", et conçoit sa célébration comme " une erreur quand elle n'est pas une manœuvre politique » (35). D'autres auteurs sont également convoqués, tels Pierre-André Taguieff et Kendall Phillips. Ce dernier, extrêmement critique à l'égard de la «culture du consensus » célébrée par Jürgen Habermas et ses successeurs, invite à prendre au sérieux les «différences constitutives des communautés " (38), des différences parfois très profondes, mais encore à ne pas renvoyer dans la déraison, ni hors du politique, ceux qui ne s'entendent pas et conservent leurs désaccords. La discussion, serrée, amène l'auteur à tracer, dans la lignée de Christian Kock (un adversaire acharné de la pragma-dialectique et de la logique informelle), les grandes lignes de sa "rhétorique du dissensus». Cette rhétorique (taillée à la mesure de l'homme) ne vise pas à persuader l'adversaire ni à gérer le conflit pour parvenir à un accord, mais bien à explorer le différend en assumant les risques qu'une telle exploration comporte. Il ne s'agit nullement de réduire au silence les parties en présence, ni d'éteindre, chez elles, la flamme incandescente de l'argumentation, mais bien de donner carrière à une démocratie pleine et entière.

9 Au titre de son travail de définition, Amossy reprend certains aspects saillants de l'excellente contribution dédiée, par Kerbrat-Orecchioni, à cet objet "fondamentalement ambivalent", la polémique donc, où des frères ennemis "s'accordent sur certains postulats idéologiques et sur certaines règles du jeu dialogique y compris le droit d'y tricher, mais discordent sur d'autres» (1980: 39). Amossy confronte alors ses vues et se positionne face aux principaux auteurs du champ : Shoshana Felman (1979), Marc Angenot (1982, 2008), Dominique Maingueneau (1983), Dominique Garand (1998), Christian Plantin (2003), Luce Albert et Loïc Nicolas (2010), etc. Le panorama qui s'en dégage clarifie les grandes tendances d'analyse et de description de la polémique, autant qu'il soulève des questions importantes relatives aux traits définitoires de celle-ci : en quel sens se distingue-t-elle du "simple débat » (55) ? Quels liens entretient-elle avec le conflit (Garand) ? Est-elle forcément violente ou, au moins, virulente, et nourrie par l'émotion vive? Implique-t-elle nécessairement une incompréhension mutuelle des participants, ainsi que des " coupures cognitives » (Angenot 2008) conduisant à un «blocage sans issue » de l'interaction verbale (58) ? Les protagonistes usent-ils toujours d'arguments ad hominem destinés à disqualifier l'adversaire? Amossy souligne, chaque fois, que les choses sont moins simples qu'il n'y paraît, et s'emploie à démêler l'essentiel de l'accessoire, la réalité du fantasme. Les cas concrets étudiés dans la suite du volume renseignent sur ce qu'est, tout compte fait, la polémique par-delà les étiquettes trop faciles qu'on essaie de lui appliquer; par-delà ses multiples caricatures.

La deuxième partie (73-139) porte attention aux «modalités discursives et argumentatives de la polémique » (73) à travers l'étude d'un double exemple relatif à la place et au statut des femmes dans l'espace public démocratique. Le premier concerne l'affaire dite de la burqa qui s'est déroulée en France entre juin 2009 et octobre 2010, tandis que le second aborde les échanges qui se sont tenus, en Israël, à la fin de l'année 
2011, à propos de l'« exclusion » et de la visibilité des femmes. Amossy se focalise, de part et d'autre, sur la sphère médiatique (au sens large), dans la mesure où c'est en son sein « que se diffuse, voire s'élabore, [la polémique] dans l'espace public » (74).

11 Peu ou prou, l'affaire de la burqa débute lorsque le 18 juin 2009 les médias commencent à s'intéresser aux discussions autour de la proposition de loi (portée par le député communiste André Guérin) d'interdire la dissimulation du visage dans l'espace public. Loi qui sera officiellement adoptée en France le 11 octobre 2010. L'étude d'Amossy s'intéresse, d'une part, à un article journalistique (signé de Bénédicte Charles et paru dans l'hebdomadaire Marianne) qui, en construisant « un dialogue agonique » à partir d'énoncés épars, a constitué la polémique «en événement dramatique et en sujet d'intérêt public » (88), d'autre part, à un débat télévisée retransmis dans le cadre de l'émission «Salut les Terriens » diffusée sur Canal+ , tout en incluant, çà et là, des références à des discours périphériques. L'analyse, très bien conduite, montre comment la polémique, «art de la réfutation » (102), opère, tout à la fois, par dichotomisation, polarisation et discrédit, mais aussi suivant des stratégies qui dépendent de la situation, donc de la configuration discursive du moment: « discours monogéré où le polémiste est seul maître à bord» (104), ou échange entre (au moins) deux débatteurs. Outre les arguments, contre-arguments, contre-contre-arguments, etc., qui se font face, réellement ou virtuellement, sont mobilisés des procédés variés (dérision, appel au bon sens ou au pathos...) destinés à mettre en péril les positions adverses. L'auteur en donne une description riche et précise.

Quoiqu'on puisse le croire, la volonté de persuader, donc le travail de persuasion, ne sont pas absents de l'entreprise polémique. Il ne s'agit pas, en ce cas, de persuader l'adversaire, du moins pas directement, mais de s'adresser au Tiers - celui qui assiste, d'une manière ou d'une autre, à l'échange polémique ; celui dont les vues, susceptibles de vaciller, sont en attente d'être confirmées, renforcées, nourries. Dès lors, Amossy souligne que, dans bien des cas, le but est, avant toute chose, de "renforcer la communauté de ceux qui se rangent dans le même camp, empêcher qu'ils ne versent dans l'indifférence et attiser leur hostilité contre la position combattue et le groupe qui la soutient " (104). Ce point, essentiel à la compréhension du phénomène polémique, échappe trop souvent aux commentateurs. Il s'agit là d'une des limites principales du travail de Marc Angenot qui, précisément, voit dans la polémique "une forme de pensée dévalorisée » (33) en raison de l'échec du processus de persuasion. Échec dont témoigne (ou témoignerait) « [l]'absence persistante de consensus » (32). Pour Angenot les polémiques interminables ne sont jamais que des « dialogues de sourds » menés par des adversaires d'idées qui, toujours, "finissent par se percevoir les uns les autres comme des "fous" "(2008: 16). Toutefois, cette "folie» dont lesdits adversaires peuvent, à l'occasion, se taxer, doit-elle être prise au pied de la lettre? Ne s'agit-il pas, tout compte fait, d'une mise en scène ou d'une ressource argumentative propre au " pacte polémique » lui-même (Albert et Nicolas 2010 : 17-48) ? Une mise en scène qui, selon Amossy, ne saurait, pour autant, être entendue comme une théâtralisation qui transformerait ipso facto la polémique en un "jeu où les participants feignent une radicalisation menant à l'impossibilité de l'accord » (226).

L'exemple israélien, très riche lui aussi, prend pour point de départ un événement (en date du 16 décembre 2011) et l'esclandre auquel il donna lieu. Cet événement, c'est, en l'occurrence, le refus d'une jeune femme (Tanya Rozenblit) de s'asseoir à l'arrière d'un bus fréquenté par la communauté ultraorthodoxe - son refus, donc, de respecter la 
coutume de séparation des sexes au sein des lignes dites mehadrin. L'auteur, sur la base d'un large corpus, analyse le " débat enflammé » (112) qui, par journaux interposés (de gauche, de droite, ultraorthodoxes), s'est fait jour quant à «l'exclusion des femmes » de l'espace public, et considère, en premier lieu, la place, les usages et l'impact de cette formule (très lourde d'implications) dans le déroulement de la polémique. La suite de l'étude donne lieu à l'exploration d'une série de procédés rhétoriques (l'héroïsation, le recours aux métaphores, à l'analogie, à l'antithèse, à l'argument de la "pente glissante ", la construction d'un "ethos collectif positif»-127, le retournement des valeurs contre l'adversaire, etc.) utilisés de part et d'autre. Amossy relève combien les polémistes - impliqués ici dans "deux ensembles de discours monogérés» (136) témoignent, par-delà le sentiment d'une incompréhension radicale, d'une véritable symétrie discursive. Symétrie suivant laquelle les propos des uns reprennent et répètent les propos des autres. La jeune Tanya Rozenblit est alors tantôt vue comme une nouvelle Rosa Parks, tantôt comme une provocatrice et une dévergondée. De fait, la polémique qui, pour reprendre une idée chère à Chantal Mouffe, convertit les " ennemis » en "adversaires ", vient créer, à travers l'excès et l'outrance, "un lien social jusque dans la polarisation" (138). Elle témoigne d'une circulation permanente des discours et des arguments. Impliqués dans cette circulation, les protagonistes apprennent à se connaître (de façon précise, bien plus précise qu'on ne pourrait le penser) et à coexister tout en s'opposant.

Les deux derniers chapitres du volume, qui composent la troisième partie (143-205), abordent certaines propriétés qu'on rattache d'ordinaire au phénomène polémique: d'abord la présence de pathos (liée, croit-on, à un manque de rationalité argumentative, à un aveuglement des protagonistes devant la cause qu'ils soutiennent), ensuite celle de violence verbale. Là encore l'auteur convoque des exemples tirés de l'actualité.

Quant à la dimension passionnelle de la polémique, Amossy prête attention au débat qui s'est tenu en France, en 2008-2009, c'est-à-dire au plus fort de la crise financière, à propos de l'attribution de bonus et de stock-options aux responsables de grandes banques et entreprises - notamment celles ayant bénéficié d'une aide de l'État français. Comme le montre l'auteur, la passion, en tant que telle, ne saurait être considérée comme la source de la polémique, elle constitue plutôt «un élan qui se veut contagieux » (152). Il n'empêche, la passion contribue, en tant que trait optionnel, à radicaliser l'échange polémique, à exacerber les tensions et la polarisation qui l'habitent. Mais cette radicalisation, née de la convocation d'éléments passionnels, ne fait pas signe vers l'irrationalité des protagonistes. Mieux, c'est le recours au pathos par exemple à l'indignation en tant que « sentiment universel» (156) - qui, justement, fonde la rationalité de l'échange même. En cela, il existe, au cœur de l'activité polémique, un jeu permanent, un va-et-vient, entre passion et raison. Analysant un article du journaliste François Sergent (directeur adjoint du quotidien Libération) intitulé «Impudence » et paru le 21 mars 2009, Amossy montre que les «qualifications dépréciatives " convoquées par l'éditorialiste viennent témoigner d'un engagement affectif qui ne s'exprime pas sans raisons. En effet, le sentiment moral est " ancré dans une conscience de la justice et une condamnation raisonnée de la transgression des normes sociales » (163). C'est pourquoi, même si ces raisons ne sont pas explicitées, même si elles restent nichées dans les interstices du discours polémique, elles signalent, en fin de compte, que « la passion joue un rôle dans la délibération publique en l'ancrant dans des valeurs et des croyances qu'un attachement affectif pousse à 
défendre jusqu'au bout, et en appelant à une condamnation morale censée modifier un état de chose jugé scandaleux » (173).

Le chapitre final, consacré aux flames dans les discussions en ligne, questionne, pour sa part, le lien, généralement posé comme évident, entre polémique et violence verbale, « violence du logos » (Kurts-Wöste, Vallespir, Watine 2013) - au sens de "parole sauvage et incontrôlée » (177). Les flames, ces manifestations d'hostilité prenant la forme de remarques incendiaires, caractérisent, bien souvent, la communication médiatisée par ordinateur. L'internet, eu égard à l'anonymat qu'il confère, en regorge, et peut donner lieu à de véritables «flambées de violence » - ce qu'on appelle alors le flaming (182). S'appuyant sur un corpus de flames tirées de forums électroniques dédiés aux bonus et aux stock-options distribués aux dirigeants d'entreprises en période de crise, Amossy pose d'emblée que si la violence ne fait pas la polémique, « on peut dire inversement (et contrairement aux idées reçues) que la polémique ne nécessite pas la violence verbale » (175). Ainsi défend-elle que la violence (possible mais pas nécessaire) des polémistes ne saurait être vue comme un renoncement à toute règle et à toute norme du discours. La brutalité, les attaques personnelles, les excès du verbe qui caractérisent les flames ne signent aucunement la sortie du champ de l'argumentation, ni de celui de la raison. Au contraire, le fait de "croiser le fer dans les discussions en ligne » constitue, pour les adversaires qui s'affrontent, une réelle occasion d'engager la discussion, fût-ce dans un échange qui ressemble " plus à une querelle qu'à un dialogue en quête d'accord» (192). En tout état de cause, la violence qui, dans le déroulement de l'échange, peut alimenter la polémique, n'a " pas pour vocation de réaliser ce qu'elle prône » (200). Fonctionnelle, cette violence est destinée à demeurer sur le strict terrain des mots - le seul légitime. Opérant par détour, elle empêche ou évite d'en venir aux mains. Admettons-le, en tant que «simulacre et substitut de la guerre littérale, les boulets» que tirent les polémistes, "aussi rouges soient-ils, ne tuent que symboliquement» (KerbratOrecchioni $1980: 6$ ). Il n'en reste pas moins qu'on ne sait pas toujours (notamment à l'heure d'internet) quelles limites assigner à la violence verbale (225). Il en va, à cet égard, de la responsabilité, de l'expérience et de la pratique des polémistes eux-mêmes.

L'ouvrage, dont il faut rappeler, encore une fois, les grandes qualités scientifiques, se referme par une conclusion très substantielle (207-228). Laquelle revient sur les moments-clés de l'enquête et synthétise les raisons qui font de la polémique, bien loin du dialogue de sourds, « un mode de coexistence dans le dissensus » (216); une façon de tisser du lien social (223) en prenant des chemins de traverse, c'est-à-dire en dérogeant aux règles trop étroites ou trop rigides de la délibération (réputée) bien conduite. Coexister de la sorte et gérer les désaccords, aussi profonds soient-ils, grâce à des ressources argumentatives ad hoc, c'est témoigner des réponses multiples, à la fois rationnelles et concurrentes, qui, toujours, se rencontrent face à un problème d'origine sociale ou politique; face aux affaires humaines en général. Moteur, ferment et condition de la vie démocratique, on comprend mieux pourquoi la polémique publique, avec « ses limites et ses défauts " (227), mérite une apologie en bonne et due forme. À cet exercice difficile Amossy s'est livrée avec courage et lucidité. D'une rare hauteur de vue, son travail est à compter, désormais, comme l'une des références majeures en la matière. 


\section{BIBLIOGRAPHY}

Albert, Luce \& Loïc Nicolas. 2010. « Le pacte polémique », Albert, Luce \& Loïc Nicolas (éds.), Polémique et rhétorique de l'Antiquité à nos jours (Bruxelles : De Boeck/Duculot, coll. « Champs linguistiques ») 17-48

Amossy, Ruth. 2010. "The Functions of Polemical Discourse in the Public Sphere ", Smith, Michelle \& Barbara Warnick (eds), The Responsibilities of Rhetoric (Long Grove : Waveland) 52-61 Amossy, Ruth. 2011. « La coexistence dans le dissensus », Amossy, Ruth \& Marcel Burger (éds.), SEMEN 31, « Polémiques médiatiques et journalistiques. La discours polémique en question(s) », $25-42$

Angenot, Marc. 1982. La Parole pamphlétaire. Typologie des discours modernes (Paris : Payot)

Angenot, Marc. 2008. Dialogues de sourds. Traité de rhétorique antilogique (Paris : Mille et Une nuits, coll. « Essais »)

Danblon, Emmanuelle. 2013. L'Homme rhétorique. Culture, raison, action (Paris : Cerf, coll. « Humanités »)

Felman, Shoshana. 1979. « Le discours polémique (Propositions préliminaires pour une théorie de la polémique) », Cahiers de l'Association internationale des études francaises, 31-1, 179-192

Garand, Dominique. 1998. « Propositions méthodologiques pour l'étude du polémique », Hayward, Annette \& Dominique Garand (éds.), États du polémique (Montréal : Nota Bene) 211-268

Garand, Dominique, Laurence Daigneault Desrosier \& Philippe Archambault. 2014. Un Québec polémique. Éthique de la discussion dans les débats publics (Montréal : Hurtubise, coll. «Cahiers du Québec : Littérature »)

Kerbrat-Orecchioni, Catherine. 1980. « La polémique et ses définitions », Kerbrat-Orecchioni, Catherine \& Nadine Gelas (éds), Le discours polémique (Lyon : PUL), 3-40

Kurts-Wöste, Lia, Mathilde Vallespir \& Marie-Albane Watine (éds), 2013. La Violence du logos. Entre sciences du texte, philosophie et littérature (Paris : Classiques Garnier, coll. «L'Univers rhétorique »)

Maingueneau, Dominique. 1983. Sémantique de la polémique. Discours religieux et ruptures idéologiques au $17^{e}$ siècle (Lausanne : L'Âge d'Homme, coll. «Cheminements »)

Perelman, Chaïm. 2009 [1945]. « Libre examen et démocratie », Modernité du libre examen, Textes de Chaïm Perelman et Jean Stengers (Bruxelles : Éd. de l’Université de Bruxelles), 51-61

Perelman, Chaïm. 1948. "Vérité contre démocratie », Cahiers du libre examen, 12-5, 7-14

Perelman, Chaïm. 1952 [1948]. « Le problème du bon choix », Perelman, Chaïm \& Lucie OlbrechtsTyteca, Rhétorique et philosophie. Pour une théorie de l'argumentation en philosophie (Paris : PUF), $142-160$

Perelman, Chaïm. 1990 [1966]. « Désaccord et rationalité des décisions », Éthique et droit (Bruxelles : Éd. de l'Université de Bruxelles), 420-428

Perelman, Chaïm. 1989 [1968]. « Une théorie philosophique de l'argumentation », Rhétoriques (Bruxelles : Éd. de l'Université de Bruxelles), 243-256

Perelman, Chaïm. 1977. L'Empire rhétorique. Rhétorique et argumentation (Paris : Vrin) 
Plantin, Christian. 2003. «Des polémistes aux polémiqueurs », Declercq, Gilles, Michel Murat \& Jacqueline Dangel (éds.), La Parole polémique (Paris : Champion), 377-408

\section{AUTHORS}

\section{LOÏC NICOLAS}

Université Libre de Bruxelles ; FNRS ; GRAL 